\title{
A Delay Almost Periodic Competitive System in Discrete Time
}

\author{
Ronghua Tan and Lvli Liao \\ Department of Mathematics, Hubei University for Nationalities, Enshi, Hubei 445000, China
}

Correspondence should be addressed to Ronghua Tan; ronghua_tan@hotmail.com

Received 12 July 2014; Accepted 17 October 2014

Academic Editor: Ryusuke Kon

Copyright (C) 2015 R. Tan and L. Liao. This is an open access article distributed under the Creative Commons Attribution License, which permits unrestricted use, distribution, and reproduction in any medium, provided the original work is properly cited.

A discrete almost periodic competitive system with delay is proposed and analyzed. The system admits a unique almost periodic solution, which is shown to be uniformly asymptotically stable by using the method of Lyapunov function. Some specific numerical examples are provided to verify our analytical results.

\section{Introduction}

As we know, a discrete time model governed by difference equations is more suitable than a corresponding continuous version when the populations have a short-life expectancy and nonoverlapping generations and can also provide efficient computation for numerical simulation (see [1-7]). On the other hand, the coefficients of model are changed owing to environmental variation. The assumption of almost periodicity of the coefficients is a way of incorporating the time-dependent variability of the environment if the various components of the environment are with incommensurable periods. One of the important ecological problems associated with the investigation of populations interaction in an almost periodic environment is the positive almost periodic solution which plays the significant role played by the equilibrium of the autonomous model (see [8-14]). In this contribution, we discuss the positive almost periodic solutions of a delay almost periodic competitive system in discrete time, and our motivation comes from the works of $[8,9,15]$.

Firstly, let us introduce the following autonomous differential model which was proposed by Ayala et al. [15]:

$$
\begin{aligned}
& x_{1}^{\prime}(t)=x_{1}(t)\left[r_{1}-x_{1}(t)-a_{1} x_{2}(t)-c_{1} x_{2}^{2}(t)\right], \\
& x_{2}^{\prime}(t)=x_{2}(t)\left[r_{2}-x_{2}(t)-a_{2} x_{1}(t)-c_{2} x_{1}^{2}(t)\right],
\end{aligned}
$$

where $x_{1}$ and $x_{2}$ stand for the population densities of two competing species. $r_{1}$ and $r_{2}$ are the intrinsic growth rates. $a_{i}$ and $c_{i}$ represent the interspecific competitive effects, $i=$ 1,2 . Assume that a species needs some time to mature and the competition occurs after some time lag required for maturity of the species; a revised version was introduced by Gopalsamy [16]

$$
\begin{aligned}
& x_{1}^{\prime}(t)=x_{1}(t)\left[r_{1}-x_{1}(t)-a_{1} x_{2}\left(t-\tau_{2}\right)-c_{1} x_{2}^{2}\left(t-\tau_{2}\right)\right], \\
& x_{2}^{\prime}(t)=x_{2}(t)\left[r_{2}-x_{2}(t)-a_{2} x_{1}\left(t-\tau_{1}\right)-c_{2} x_{1}^{2}\left(t-\tau_{1}\right)\right] .
\end{aligned}
$$

Furthermore, considering the biological parameters naturally being subject to almost periodic fluctuation in time and the influence of many generations on the density of species population, we establish the following model:

$$
\begin{aligned}
& \begin{aligned}
x_{1}(n+1) & \\
=x_{1}(n) \exp [ & r_{1}(n)-x_{1}(n) \\
& \left.\quad-a_{1}(n) x_{2}\left(n-\tau_{2}\right)-c_{1}(n) x_{2}^{2}\left(n-\tau_{2}\right)\right], \\
x_{2}(n+1) \quad & \\
=x_{2}(n) \exp [ & r_{2}(n)-x_{2}(n) \\
& \left.\quad-a_{2}(n) x_{1}\left(n-\tau_{1}\right)-c_{2}(n) x_{1}^{2}\left(n-\tau_{1}\right)\right],
\end{aligned}
\end{aligned}
$$


$n=0,1,2, \ldots$, and the initial conditions satisfy

$$
\begin{aligned}
x_{1}(\theta)=\phi_{1}(\theta) \geq 0, & x_{2}(\theta)=\phi_{2}(\theta) \geq 0, \\
\phi_{1}(0)>0, & \phi_{2}(0)>0, \\
\theta \in\{-\tau,-\tau+1, \ldots, 0\}, & \tau=\max \left\{\tau_{i}, i=1,2\right\} .
\end{aligned}
$$

Here $x_{i}(n)$ and $r_{i}(n)$ are, respectively, the densities and intrinsic growth rates of species $x_{i}$ at the $n$th generation. $a_{i}(n)$ and $c_{i}(n)$ measure the interspecific influence of the $\left(n-\tau_{j}\right)$ th generation of species $x_{j}$ on species $x_{i}(i, j=1,2 ; i \neq j)$. The delays $\tau_{1}$ and $\tau_{2}$ are positive integers and the coefficients $\left\{r_{i}(n)\right\},\left\{a_{i}(n)\right\}$, and $\left\{c_{i}(n)\right\}$ are bounded positive almost periodic sequences, $i=1,2$.

The rest part of this paper is organized as follows. In Section 2, some preliminaries are given. Sufficient conditions for the uniformly asymptotic stability of a unique positive almost periodic solution for the system are established in Section 3. In final section, some specific numerical examples are carried out to illustrate the feasibilities of our theoretical results.

\section{Preliminaries}

This section is concerned with some notations, definitions, and lemmas which will be used for our main results.

Let $R, R^{+}, Z$, and $Z^{+}$be, respectively, the sets of real numbers, nonnegative real numbers, integers, and nonnegative integers. Assign $R^{2}$ and $R^{k}$ which denote the cone of 2 dimensional and $k$-dimensional real Euclidean space, respectively. Denote $[a, b]_{Z}=[a, b] \bigcap Z, a, b \in Z$, and then $K=$ $[-\tau,+\infty)_{Z}$, where $\tau$ is defined in (4). For simplicity in the following discussion, we use the notations $\mathscr{A}^{U}=\sup _{n \in Z^{+}}\{\mathscr{A}(n)\}$, $\mathscr{A}^{L}=\inf _{n \in Z^{+}}\{\mathscr{A}(n)\}$, where $\{\mathscr{A}(n)\}$ is an almost periodic sequence.

Definition 1 (see [12]). A sequence $x: Z \rightarrow R^{k}$ is called an almost periodic sequence if the $\varepsilon$-translation set of $x$

$$
E\{\varepsilon, x\}:=\{\tau \in Z:|x(n+\tau)-x(n)|<\varepsilon, \forall n \in Z\}
$$

is a relatively dense set in $Z$ for all $\varepsilon>0$, that is, for any given $\varepsilon>0$, there exists an integer $l(\varepsilon)>0$ such that each discrete interval of length $l(\varepsilon)$ contains an integer $\tau=\tau(\varepsilon) \in E\{\varepsilon, x\}$ such that $|x(n+\tau)-x(n)|<\varepsilon, \forall n \in Z$. $\tau$ is called the $\varepsilon^{-}$ translation number of $x(n)$.

Definition 2 (see [12]). Let $f: Z \times D \rightarrow R^{k}$, where $D$ is an open set in $C:\left\{\varphi:[-\tau, 0]_{Z} \rightarrow R^{k}\right\} . f(n, \varphi)$ is said to be almost periodic in $n$ uniformly for $\varphi \in D$, if for any $\varepsilon>0$ and any compact set $S$ in $D$, there exists a positive integer $l(\varepsilon, S)$ such that any interval of length $l(\varepsilon, S)$ contains an integer $\tau$ for which

$$
|f(n+\tau, \varphi)-f(n, \varphi)|<\varepsilon, \quad \forall n \in Z, \varphi \in S,
$$

and $\tau$ is called the $\varepsilon$-translation number of $f(n, \varphi)$.

Lemma 3 (see [17]). $\{x(n)\}$ is an almost periodic sequence if and only iffor any sequence $\left\{t_{k}^{\prime}\right\} \subset Z$ there exists a subsequence $\left\{t_{k}\right\} \subset\left\{t_{k}^{\prime}\right\}$ such that $x\left(n+t_{k}\right)$ converges uniformly on $n \in Z$ as $k \rightarrow+\infty$.

Zhang and Zheng [12] considered the following almost periodic delay difference system:

$$
x(n+1)=f\left(n, x_{n}\right), \quad n \in Z^{+},
$$

where $f: Z^{+} \times C_{B} \rightarrow R^{k}, C_{B}=\{\varphi \in C:\|\varphi\|<B\}, C=$ $\left\{\varphi:[-\tau, 0]_{Z} \rightarrow R^{k}\right\}$ with $\|\varphi\|=\sup _{s \in[-\tau, 0]_{Z}}|\varphi(s)|, f(n, \varphi)$ is almost periodic in $n$ uniformly for $\varphi \in C_{B}$ and is continuous in $\varphi$, while $x_{n} \in C_{B}$ is defined as $x_{n}(s)=x(n+s)$ for all $s \in[-\tau, 0]_{Z}$. The product system of $(7)$ is as follows:

$$
x(n+1)=f\left(n, x_{n}\right), \quad y(n+1)=f\left(n, y_{n}\right) .
$$

A discrete Lyapunov function of (8) is a function $V: Z^{+} \times$ $C_{B} \times C_{B} \rightarrow R^{+}$which is continuous in its second and third variables. Define the difference of $V$ along the solution of system (8) by

$$
\begin{aligned}
& \Delta V_{(8)}(n, \varphi, \psi) \\
& \quad=V\left(n+1, x_{n+1}(n, \varphi), y_{n+1}(n, \psi)\right)-V(n, \varphi, \psi),
\end{aligned}
$$

where $(x(n, \varphi), y(n, \psi))$ is a solution of system (8) through $(n,(\varphi, \psi)), \varphi, \psi \in C_{B}$.

Lemma 4 (see [12]). Suppose that there exists a Lyapunov function $V(n, \varphi, \psi)$ satisfying the following conditions.

(I) $a(|\varphi(0)-\psi(0)|) \leq V(n, \varphi, \psi) \leq b(\|\varphi-\psi\|)$, where $a, b \in$ $P$ with $P=\{a:[0,+\infty) \rightarrow[0,+\infty) \mid a(0)=0$ and $a(u)$ is continuous, increasing in $u\}$.

(II) $\left|V\left(n, \varphi_{1}, \psi_{1}\right)-V\left(n, \varphi_{2}, \psi_{2}\right)\right| \leq L\left(\left\|\varphi_{1}-\varphi_{2}\right\|+\left\|\psi_{1}-\psi_{2}\right\|\right)$, where $L>0$ is a constant.

(III) $\Delta V(n, \varphi, \psi) \leq-\alpha V(n, \varphi, \psi)$, where $0<\alpha<1$ is a constant.

Moreover, if there exists a solution $x(n)$ of system (7) such that $\left\|x_{n}\right\| \leq B^{*} \leq B$ for all $n \in Z^{+}$, then there exists a unique uniformly asymptotically stable almost periodic solution $q(n)$ of system (7) which satisfies $|q(n)| \leq B^{*}$ for all $n \in K$. In particular, if $f(n, \varphi)$ is periodic of period $\omega$, then system (7) has a unique uniformly asymptotically stable periodic solution of period $\omega$.

Remark 5 (see [9]). Condition (III) of Lemma 4 can be replaced by

(III) $)^{\prime} \Delta V(n, \varphi, \psi) \leq-\beta(|\varphi(0)-\psi(0)|)$, where $\beta \in\{c$ : $[0,+\infty) \rightarrow[0,+\infty) \mid c$ is continuous, $c(0)=0$ and $c(s)>0$ for $s>0\}$.

Lemma 6 (see $[18])$. Assume that $x(n)>0$ and

$$
\begin{array}{r}
x(n+1) \leq x(n) \exp (r(n)(1-a x(n))) \\
\text { for } n \in\left[n_{1},+\infty\right),
\end{array}
$$

where $r(n)$ is a bounded positive sequence and $a$ is a positive constant. Then

$$
\limsup _{n \rightarrow+\infty} x(n) \leq \frac{1}{a r^{U}} \exp \left(r^{U}-1\right) .
$$


Lemma 7 (see [18]). Assume that $\{x(n)\}$ satisfies

$$
x(n+1) \geq x(n) \exp (r(n)(1-a x(n))), \quad n \geq N_{0},
$$

$\limsup _{n \rightarrow+\infty} x(n) \leq \mathscr{M}$ and $x\left(N_{0}\right)>0$, where $r(n)$ is a bounded positive sequence and $a$ is a positive constant such that a. $>1$ and $N_{0} \in Z^{+}$. Then

$$
\liminf _{n \rightarrow+\infty} x(n) \geq \frac{1}{a} \exp \left(r^{U}(1-a \mathscr{M})\right) .
$$

Denote

$$
\begin{aligned}
& H_{i}=\exp \left(r_{i}^{U}-1\right), \quad \Delta_{i}=r_{i}^{L}-a_{i}^{U} H_{j}-c_{i}^{U} H_{j}^{2}, \\
& h_{i}=\Delta_{i} \exp \left(\Delta_{i}-H_{i}\right), \quad i, j=1,2 ; i \neq j .
\end{aligned}
$$

Lemma 8. If $\Delta_{i}>0$, then any positive solution $\left(x_{1}(n)\right.$, $\left.x_{2}(n)\right)$ of system (3) satisfies $h_{i} \leq \liminf _{n \rightarrow+\infty} x_{i}(n) \leq$ $\lim \sup _{n \rightarrow+\infty} x_{i}(n) \leq H_{i}, i=1,2$.

Proof. According to the first equation of system (3), one has

$$
\begin{aligned}
x_{1}(n+1) & \leq x_{1}(n) \exp \left[r_{1}(n)-x_{1}(n)\right] \\
& \leq x_{1}(n)\left[r_{1}(n)\left(1-\frac{1}{r_{1}^{U}} x_{1}(n)\right)\right] .
\end{aligned}
$$

It follows from Lemma 6 that

$$
\limsup _{n \rightarrow+\infty} x_{1}(n) \leq \exp \left(r_{1}^{U}-1\right)=H_{1} \text {. }
$$

Analogously, from the second equation of system (3) we obtain that

$$
\limsup _{n \rightarrow+\infty} x_{2}(n) \leq \exp \left(r_{2}^{U}-1\right)=H_{2} .
$$

Assigning a positive constant $\varepsilon$ arbitrarily small, it follows from (16) and (17) that there exists a large enough $n_{1}>0$ such that for all $n \geq n_{1}$,

$$
x_{i}(n) \leq H_{i}+\varepsilon, \quad i=1,2 .
$$

From the first equation of system (3), for $n \geq n_{1}+\tau$,

$$
\begin{aligned}
& x_{1}(n+1) \\
& \geq x_{1}(n) \exp \left[r_{1}^{L}-x_{1}(n)-a_{1}^{U}\left(H_{2}+\varepsilon\right)-c_{1}^{U}\left(H_{2}+\varepsilon\right)^{2}\right] \\
& =x_{1}(n) \exp \left[\left(r_{1}^{L}-a_{1}^{U}\left(H_{2}+\varepsilon\right)-c_{1}^{U}\left(H_{2}+\varepsilon\right)^{2}\right)\right. \\
& \left.\quad \times\left(1-\frac{x_{1}(n)}{r_{1}^{L}-a_{1}^{U}\left(H_{2}+\varepsilon\right)-c_{1}^{U}\left(H_{2}+\varepsilon\right)^{2}}\right)\right] .
\end{aligned}
$$

Using Lemma 7 , for $n \geq n_{1}+\tau$, one has

$$
\begin{aligned}
& \liminf _{n \rightarrow+\infty} x_{1}(n) \\
& \geq\left(r_{1}^{L}-a_{1}^{U}\left(H_{2}+\varepsilon\right)-c_{1}^{U}\left(H_{2}+\varepsilon\right)^{2}\right) \\
& \quad \times \exp \left[r_{1}^{L}-a_{1}^{U}\left(H_{2}+\varepsilon\right)-c_{1}^{U}\left(H_{2}+\varepsilon\right)^{2}-H_{1}\right] .
\end{aligned}
$$

Setting $\varepsilon \rightarrow 0$, it follows that

$$
\begin{aligned}
\liminf _{n \rightarrow+\infty} x_{1}(n) \geq & \left(r_{1}^{L}-a_{1}^{U} H_{2}-c_{1}^{U} H_{2}^{2}\right) \\
& \times \exp \left[r_{1}^{L}-a_{1}^{U} H_{2}-c_{1}^{U} H_{2}^{2}-H_{1}\right]=h_{1} .
\end{aligned}
$$

Similar to the above argument, from the second equation of system (3) we can obtain that

$$
\begin{aligned}
\liminf _{n \rightarrow+\infty} x_{2}(n) \geq & \left(r_{2}^{L}-a_{2}^{U} H_{1}-c_{2}^{U} H_{1}^{2}\right) \\
& \times \exp \left[r_{2}^{L}-a_{2}^{U} H_{1}-c_{2}^{U} H_{1}^{2}-H_{2}\right]=h_{2} .
\end{aligned}
$$

This completes the proof.

Denote $\Theta$ by

$$
\begin{gathered}
\Theta=\{\text { every solution of system (3) satisfying } \\
\left.h_{i} \leq x_{i}(n) \leq H_{i}, \quad i=1,2 \forall n \in K\right\} .
\end{gathered}
$$

Lemma 9. If $\Delta_{i}>0$, then $\Theta \neq \emptyset$, where $\Delta_{i}$ are defined in (14).

Proof. Since $\left\{r_{i}(n)\right\},\left\{a_{i}(n)\right\}$, and $\left\{c_{i}(n)\right\}, i=1,2$ are almost periodic sequences, there exists a positive integer sequence $\left\{t_{k}\right\}$ with $t_{k} \rightarrow+\infty$ as $k \rightarrow+\infty$ such that

$$
\begin{array}{r}
r_{i}\left(n+t_{k}\right) \longrightarrow r_{i}(n), \quad a_{i}\left(n+t_{k}\right) \longrightarrow a_{i}(n), \\
c_{i}\left(n+t_{k}\right) \longrightarrow c_{i}(n), \quad i=1,2,
\end{array}
$$

as $k \rightarrow+\infty$ for $n \in Z^{+}$. By (16), (17), (21), and (22), for any sufficiently small $\varepsilon>0$, there exists a positive integer $n_{0}$ such that for all $n>n_{0}$,

$$
h_{i}-\varepsilon \leq x_{i}(n) \leq H_{i}+\varepsilon .
$$

Assign

$$
x_{i k}(n)=x_{i}\left(n+t_{k}\right) \quad \text { for } n \geq n_{0}+\tau-t_{k}, k=1,2, \ldots
$$

For any positive integer $q$, it is obvious that there exists a sequence $\left\{x_{i k}(n): k \geq q\right\}$ such that the sequence $\left\{x_{i k}(n)\right\}$ has a subsequence, also denoted by $\left\{x_{i k}(n)\right\}$, converging on any definite interval of $K$ as $k \rightarrow+\infty$. Thus, there is a sequence $\left\{w_{i}(n)\right\}$ satisfying

$$
x_{i k}(n) \longrightarrow w_{i}(n), \quad \text { for } n \in K \text { as } k \longrightarrow+\infty,
$$

which, together with (24), yields that

$$
\begin{aligned}
x_{1 k}(n+1)=x_{1 k}(n) \exp [ & r_{1}\left(n+t_{k}\right)-x_{1 k}(n) \\
& -a_{1}\left(n+t_{k}\right) x_{2 k}\left(n-\tau_{2}\right) \\
& \left.-c_{1}\left(n+t_{k}\right) x_{2 k}^{2}\left(n-\tau_{2}\right)\right], \\
x_{2 k}(n+1)=x_{2 k}(n) \exp [ & r_{2}\left(n+t_{k}\right)-x_{2 k}(n) \\
& -a_{2}\left(n+t_{k}\right) x_{1 k}\left(n-\tau_{1}\right) \\
& \left.-c_{2}\left(n+t_{k}\right) x_{1 k}^{2}\left(n-\tau_{1}\right)\right],
\end{aligned}
$$


we have from (24), (27), and (28) that

$$
\begin{aligned}
w_{1}(n+1)=w_{1}(n) \exp [ & r_{1}(n)-w_{1}(n) \\
& -a_{1}(n) w_{2}\left(n-\tau_{2}\right) \\
& \left.-c_{1}(n) w_{2}^{2}\left(n-\tau_{2}\right)\right], \\
w_{2}(n+1)=w_{2}(n) \exp [ & r_{2}(n)-w_{2}(n) \\
& -c_{2}(n) w_{1}\left(n-\tau_{1}\right) \\
& \left.-c_{2}(n) w_{1}^{2}\left(n-\tau_{1}\right)\right] .
\end{aligned}
$$

It is easy to see that $\left(w_{1}(n), w_{2}(n)\right)$ is a solution of system (3) and $h_{i}-\varepsilon \leq w_{i}(n) \leq H_{i}+\varepsilon$ for $n \in K$. Since $\varepsilon$ is small enough, we derive that $h_{i} \leq w_{i}(n) \leq H_{i}, i=1,2$ for $n \in K$. This completes the proof.

\section{Main Result}

In this section, we focus on the result of the uniformly asymptotic stability of positive almost periodic solutions of system (3).

Denote

$$
\begin{aligned}
\lambda_{i}= & 1-\max \left(\left|1-h_{i}\right|,\left|1-H_{i}\right|\right) \\
& -\left(a_{j}^{U} H_{i}+2 c_{j}^{U} H_{i}^{2}\right), \quad i, j=1,2 ; i \neq j .
\end{aligned}
$$

Theorem 10. If $\Delta_{i}>0$ and $\lambda_{i}>0$, system (3) has a unique positive almost periodic solution which is uniformly asymptotically stable, where $\Delta_{i}$ are defined in (14).

Proof. We make the change of variables

$$
y_{1}(n)=\ln x_{1}(n), \quad y_{2}(n)=\ln x_{2}(n),
$$

and then system (3) can be rewritten as

$$
\begin{aligned}
y_{1}(n+1)= & y_{1}(n)+r_{1}(n)-e^{y_{1}(n)} \\
& -a_{1}(n) e^{y_{2}\left(n-\tau_{2}\right)}-c_{1}(n) e^{2 y_{2}\left(n-\tau_{2}\right)}, \\
y_{2}(n+1)= & y_{2}(n)+r_{2}(n)-e^{y_{2}(n)} \\
& -a_{2}(n) e^{y_{1}\left(n-\tau_{1}\right)}-c_{2}(n) e^{2 y_{1}\left(n-\tau_{1}\right)} .
\end{aligned}
$$

It follows from Lemma 9 that there exists a bounded solution $\left(y_{1}(n), y_{2}(n)\right)$ of system (32) satisfying

$$
\begin{array}{r}
\ln h_{1} \leq y_{1}(n) \leq \ln H_{1}, \quad \ln h_{2} \leq y_{2}(n) \leq \ln H_{2}, \\
\forall n \in K,
\end{array}
$$

which implies that $\left|y_{1}(n)\right| \leq B_{1},\left|y_{2}(n)\right| \leq B_{2}$, where $B_{i}=$ $\max \left\{\left|\ln h_{i}\right|,\left|\ln H_{i}\right|\right\}, i=1,2$. Assign

$$
\begin{array}{r}
Y_{n}(s)=\left(y_{1}(n+s), y_{2}(n+s)\right), \\
Z_{n}(s)=\left(z_{1}(n+s), z_{2}(n+s)\right), \\
n \in Z^{+}, \quad s \in[-\tau, 0]_{Z}
\end{array}
$$

are two solutions of system (32) defined on $D$, where

$$
\begin{aligned}
D=\left\{\left(y_{1}(n), y_{2}(n)\right) \mid\right. & \\
& \left.\ln h_{i} \leq y_{i}(n) \leq \ln H_{i}, i=1,2, n \in K\right\} .
\end{aligned}
$$

Definning

$$
\begin{aligned}
\left\|Y_{n}(s)\right\| & =\left\|\left(y_{1}(n+s), y_{2}(n+s)\right)\right\| \\
& =\sup _{s \in[-\tau, 0]_{Z}}\left\{\left|y_{1}(n+s)\right|+\left|y_{2}(n+s)\right|\right\},
\end{aligned}
$$

where $\left(y_{1}(n+s), y_{2}(n+s)\right) \in R^{2}$, we have

$$
\left\|Y_{n}\right\| \leq A, \quad\left\|Z_{n}\right\| \leq A
$$

where $A=B_{1}+B_{2}$.

Let us consider the associate product system of system (32)

$$
\begin{aligned}
y_{1}(n+1)= & y_{1}(n)+r_{1}(n)-e^{y_{1}(n)} \\
& -a_{1}(n) e^{y_{2}\left(n-\tau_{2}\right)}-c_{1}(n) e^{2 y_{2}\left(n-\tau_{2}\right)}, \\
y_{2}(n+1)= & y_{2}(n)+r_{2}(n)-e^{y_{2}(n)} \\
& -a_{2}(n) e^{y_{1}\left(n-\tau_{1}\right)}-c_{2}(n) e^{2 y_{1}\left(n-\tau_{1}\right)}, \\
z_{1}(n+1)= & z_{1}(n)+r_{1}(n)-e^{z_{1}(n)} \\
& -a_{1}(n) e^{z_{2}\left(n-\tau_{2}\right)}-c_{1}(n) e^{2 z_{2}\left(n-\tau_{2}\right)}, \\
z_{2}(n+1)= & z_{2}(n)+r_{2}(n)-e^{z_{2}(n)} \\
& -a_{2}(n) e^{z_{1}\left(n-\tau_{1}\right)}-c_{2}(n) e^{2 z_{1}\left(n-\tau_{1}\right)} .
\end{aligned}
$$

Construct the following Lyapunov function $V(n)=V\left(n, Y_{n}\right.$, $Z_{n}$ ) defined on $Z^{+} \times D \times D$

$$
\begin{aligned}
V(n)= & V\left(n, Y_{n}, Z_{n}\right)=\left|y_{1}(n)-z_{1}(n)\right|+\left|y_{2}(n)-z_{2}(n)\right| \\
& +\sum_{\zeta=n-\tau_{1}}^{n-1}\left(a_{2}^{U} H_{1}+2 c_{2}^{U} H_{1}^{2}\right)\left|y_{1}(\zeta)-z_{1}(\zeta)\right| \\
& +\sum_{\zeta=n-\tau_{2}}^{n-1}\left(a_{1}^{U} H_{2}+2 c_{1}^{U} H_{2}^{2}\right)\left|y_{2}(\zeta)-z_{2}(\zeta)\right|
\end{aligned}
$$


Apparently,

$$
\begin{aligned}
\left|Y_{n}(0)-Z_{n}(0)\right| & \\
\leq & V(n) \leq\left|y_{1}(n)-z_{1}(n)\right|+\left|y_{2}(n)-z_{2}(n)\right| \\
& +\sum_{\zeta=n-\tau}^{n-1}\left(a_{2}^{U} H_{1}+2 c_{2}^{U} H_{1}^{2}\right)\left|y_{1}(\zeta)-z_{1}(\zeta)\right| \\
& +\sum_{\zeta=n-\tau}^{n-1}\left(a_{1}^{U} H_{2}+2 c_{1}^{U} H_{2}^{2}\right)\left|y_{2}(\zeta)-z_{2}(\zeta)\right| \\
\leq & \left|y_{1}(n)-z_{1}(n)\right|+\left|y_{2}(n)-z_{2}(n)\right| \\
& +\sum_{\zeta=n-\tau}^{n-1} \sigma\left\{\left|y_{1}(\zeta)-z_{1}(\zeta)\right|+\left|y_{2}(\zeta)-z_{2}(\zeta)\right|\right\} \\
\leq & (1+\tau \sigma) \sup _{s \in[-\tau, 0]_{z}}\left\{\left|y_{1}(n+s)-z_{1}(n+s)\right|\right. \\
= & \left.\rho\left\|Y_{n}-Z_{n}\right\|, \quad+\left|y_{2}(n+s)-z_{2}(n+s)\right|\right\}
\end{aligned}
$$

where

$$
\begin{gathered}
\left|Y_{n}(0)-Z_{n}(0)\right| \\
\quad=\left[\left(y_{1}(n)-z_{1}(n)\right)^{2}+\left(y_{2}(n)-z_{2}(n)\right)^{2}\right]^{1 / 2} \\
\leq\left|y_{1}(n)-z_{1}(n)\right|+\left|y_{2}(n)-z_{2}(n)\right|, \\
\rho=1+\tau \sigma, \quad \sigma=\max \left\{a_{2}^{U} H_{1}+2 c_{2}^{U} H_{1}^{2}, a_{1}^{U} H_{2}+2 c_{1}^{U} H_{2}^{2}\right\} .
\end{gathered}
$$

\section{Denote}

$$
a(x)=x, \quad b=\rho x, \quad a, b \in C\left(R^{+}, R^{+}\right),
$$

and thus condition (I) in Lemma 4 is satisfied.

$$
\text { For any } y, z, \bar{y}, \bar{z} \text {, we obtain that }
$$

$$
\begin{aligned}
\| y & -z|-| \bar{y}-\bar{z}|| \\
& = \begin{cases}|y-z|-|\bar{y}-\bar{z}|, & \text { if }|y-z| \geq|\bar{y}-\bar{z}|, \\
|\bar{y}-\bar{z}|-|y-z|, & \text { if }|\bar{y}-\bar{z}|>|y-z|\end{cases} \\
& \leq \begin{cases}|(y-\bar{y})+(\bar{z}-z)|, & \text { if }|y-z| \geq|\bar{y}-\bar{z}|, \\
\mid \bar{y}-y)+(z-\bar{z}) \mid, & \text { if }|\bar{y}-\bar{z}|>|y-z|\end{cases} \\
& \leq \begin{cases}|y-\bar{y}|+|\bar{z}-z|, & \text { if }|y-z| \geq|\bar{y}-\bar{z}|, \\
|\bar{y}-y|+|z-\bar{z}|, & \text { if }|\bar{y}-\bar{z}|>|y-z|\end{cases} \\
& =|y-\bar{y}|+|z-\bar{z}| .
\end{aligned}
$$

Hence, for any $Y_{n}, Z_{n}, \bar{Y}_{n}, \bar{Z}_{n} \in D$, we obtain, by (44), that

$$
\begin{aligned}
\left|V\left(n, Y_{n}, Z_{n}\right)-V\left(n, \bar{Y}_{n}, \bar{Z}_{n}\right)\right| \\
=\left|y_{1}(n)-z_{1}(n)\right|+\left|y_{2}(n)-z_{2}(n)\right| \\
\quad+\sum_{\zeta=n-\tau_{1}}^{n-1}\left(a_{2}^{U} H_{1}+2 c_{2}^{U} H_{1}^{2}\right)\left|y_{1}(\zeta)-z_{1}(\zeta)\right| \\
\quad+\sum_{\zeta=n-\tau_{2}}^{n-1}\left(a_{1}^{U} H_{2}+2 c_{1}^{U} H_{2}^{2}\right)\left|y_{2}(\zeta)-z_{2}(\zeta)\right| \\
\quad-\left|\bar{y}_{1}(n)-\bar{z}_{1}(n)\right|-\left|\bar{y}_{2}(n)-\bar{z}_{2}(n)\right| \\
\quad-\sum_{\zeta=n-\tau_{1}}^{n-1}\left(a_{2}^{U} H_{1}+2 c_{2}^{U} H_{1}^{2}\right)\left|\bar{y}_{1}(\zeta)-\bar{z}_{1}(\zeta)\right| \\
\quad-\sum_{\zeta=n-\tau_{2}}^{n-1}\left(a_{1}^{U} H_{2}+2 c_{1}^{U} H_{2}^{2}\right)\left|\bar{y}_{2}(\zeta)-\bar{z}_{2}(\zeta)\right|
\end{aligned}
$$$$
\leq|| y_{1}(n)-z_{1}(n)|+| y_{2}(n)-z_{2}(n) \mid
$$$$
-\left|\bar{y}_{1}(n)-\bar{z}_{1}(n)\right|-\left|\bar{y}_{2}(n)-\bar{z}_{2}(n)\right| \mid
$$$$
+\sum_{\zeta=n-\tau_{1}}^{n-1}\left(a_{2}^{U} H_{1}+2 c_{2}^{U} H_{1}^{2}\right)
$$$$
\times\left\|y_{1}(\zeta)-z_{1}(\zeta)|-| \bar{y}_{1}(\zeta)-\bar{z}_{1}(\zeta)\right\|
$$$$
+\sum_{\zeta=n-\tau_{2}}^{n-1}\left(a_{1}^{U} H_{2}+2 c_{1}^{U} H_{2}^{2}\right)
$$$$
\times|| y_{2}(\zeta)-z_{2}(\zeta)|-| \bar{y}_{2}(\zeta)-\bar{z}_{2}(\zeta) \|
$$

$$
\leq\left\{\left|y_{1}(n)-\bar{y}_{1}(n)\right|+\left|y_{2}(n)-\bar{y}_{2}(n)\right|\right.
$$

$$
\left.+\left|z_{1}(n)-\bar{z}_{1}(n)\right|+\left|z_{2}(n)-\bar{z}_{2}(n)\right|\right\}
$$

$$
+\sum_{\zeta=n-\tau}^{n-1}\left(a_{2}^{U} H_{1}+2 c_{2}^{U} H_{1}^{2}\right)
$$

$$
\times\left\{\left|y_{1}(\zeta)-\bar{y}_{1}(\zeta)\right|+\left|z_{1}(\zeta)-\bar{z}_{1}(\zeta)\right|\right\}
$$

$$
\begin{aligned}
+\sum_{\zeta=n-\tau}^{n-1}\left(a_{1}^{U} H_{2}+2 c_{1}^{U} H_{2}^{2}\right) \\
\quad \times\left\{\left|y_{2}(\zeta)-\bar{y}_{2}(\zeta)\right|+\left|z_{2}(\zeta)-\bar{z}_{2}(\zeta)\right|\right\}
\end{aligned}
$$

$$
\leq\left\{\left|y_{1}(n)-\bar{y}_{1}(n)\right|+\left|y_{2}(n)-\bar{y}_{2}(n)\right|\right.
$$$$
\left.+\left|z_{1}(n)-\bar{z}_{1}(n)\right|+\left|z_{2}(n)-\bar{z}_{2}(n)\right|\right\}
$$$$
+\sum_{\zeta=n-\tau}^{n-1} \sigma\left\{\left|y_{1}(\zeta)-\bar{y}_{1}(\zeta)\right|+\left|z_{1}(\zeta)-\bar{z}_{1}(\zeta)\right|\right.
$$

$$
\left.+\left|y_{2}(\zeta)-\bar{y}_{2}(\zeta)\right|+\left|z_{2}(\zeta)-\bar{z}_{2}(\zeta)\right|\right\}
$$




$$
\begin{aligned}
& \leq(1+\tau \sigma) \sup _{s \in[-\tau, 0]_{Z}}\{\mid y_{1}(n+s)-\bar{y}_{1}(n+s) \mid \\
&+\left|y_{2}(n+s)-\bar{y}_{1}(n+s)\right| \\
&+\left|z_{1}(n+s)-\bar{z}_{1}(n+s)\right| \\
&\left.+\left|z_{2}(n+s)-\bar{z}_{2}(n+s)\right|\right\} \\
& \leq \rho\left(\left\|Y_{n}-\bar{Y}_{n}\right\|+\left\|Z_{n}-\bar{Z}_{n}\right\|\right),
\end{aligned}
$$

where $\rho$ and $\sigma$ are defined in (42). That is, condition (II) in Lemma 4 is also satisfied.

It follows from the mean-value theorem that we find

$$
\begin{gathered}
e^{y_{i}(n)}-e^{z_{i}(n)}=e^{\theta_{i}(n)}\left(y_{i}(n)-z_{i}(n)\right), \\
e^{y_{i}\left(n-\tau_{i}\right)}-e^{z_{i}\left(n-\tau_{i}\right)}=e^{\eta_{i}\left(n-\tau_{i}\right)}\left(y_{i}\left(n-\tau_{i}\right)-z_{i}\left(n-\tau_{i}\right)\right), \\
e^{2 y_{i}\left(n-\tau_{i}\right)}-e^{2 z_{i}\left(n-\tau_{i}\right)} \\
=2 e^{2 \varsigma_{i}\left(n-\tau_{i}\right)}\left(y_{i}\left(n-\tau_{i}\right)-z_{i}\left(n-\tau_{i}\right)\right), \quad i=1,2,
\end{gathered}
$$

where $\theta_{i}(n)$ lie between $y_{i}(n)$ and $z_{i}(n)$ and $\eta_{i}\left(n-\tau_{i}\right), \varsigma_{i}\left(n-\tau_{i}\right)$ all lie between $y_{i}\left(n-\tau_{i}\right)$ and $z_{i}\left(n-\tau_{i}\right)$, respectively. Then

$$
\ln h_{i} \leq \theta_{i}(n), \quad \eta_{i}\left(n-\tau_{i}\right), \quad \varsigma_{i}\left(n-\tau_{i}\right) \leq \ln H_{i}
$$

$n \in Z^{+}$.

This one together with system (38) and (46) yields that

$$
\begin{aligned}
\left|y_{1}(n+1)-z_{1}(n+1)\right|+\left|y_{2}(n+1)-z_{2}(n+1)\right| \\
=\mid y_{1}(n)-z_{1}(n)-e^{\theta_{1}(n)}\left(y_{1}(n)-z_{1}(n)\right) \\
\quad-a_{1}(n) e^{\eta_{2}\left(n-\tau_{2}\right)}\left(y_{2}\left(n-\tau_{2}\right)-z_{2}\left(n-\tau_{2}\right)\right) \\
\quad-2 c_{1}(n) e^{2 \varsigma_{2}\left(n-\tau_{2}\right)}\left(y_{2}\left(n-\tau_{2}\right)-z_{2}\left(n-\tau_{2}\right)\right) \mid \\
+\mid y_{2}(n)-z_{2}(n)-e^{\theta_{2}(n)}\left(y_{2}(n)-z_{2}(n)\right) \\
\quad-a_{2}(n) e^{\eta_{1}\left(n-\tau_{1}\right)}\left(y_{1}\left(n-\tau_{1}\right)-z_{1}\left(n-\tau_{1}\right)\right) \\
\quad-2 c_{2}(n) e^{2 \varsigma_{1}\left(n-\tau_{1}\right)}\left(y_{1}\left(n-\tau_{1}\right)-z_{1}\left(n-\tau_{1}\right)\right) \mid \\
\leq\left|1-e^{\theta_{1}(n)}\right| \cdot\left|y_{1}(n)-z_{1}(n)\right| \\
+\left(a_{1}(n) e^{\eta_{2}\left(n-\tau_{2}\right)}+2 c_{1}(n) e^{2 \varsigma_{2}\left(n-\tau_{2}\right)}\right) \\
\times\left|y_{2}\left(n-\tau_{2}\right)-z_{2}\left(n-\tau_{2}\right)\right| \\
+\left|1-e^{\theta_{2}(n)}\right| \cdot\left|y_{2}(n)-z_{2}(n)\right| \\
+\left(a_{2}(n) e^{\eta_{1}\left(n-\tau_{1}\right)}+2 c_{2}(n) e^{2 \varsigma_{1}\left(n-\tau_{1}\right)}\right) \\
\times\left|y_{1}\left(n-\tau_{1}\right)-z_{1}\left(n-\tau_{1}\right)\right|
\end{aligned}
$$

$$
\begin{aligned}
\leq & \left|1-e^{\theta_{1}(n)}\right| \cdot\left|y_{1}(n)-z_{1}(n)\right| \\
& +\left(a_{1}^{U} H_{2}+2 c_{1}^{U} H_{2}^{2}\right)\left|y_{2}\left(n-\tau_{2}\right)-z_{2}\left(n-\tau_{2}\right)\right| \\
& +\left|1-e^{\theta_{2}(n)}\right| \cdot\left|y_{2}(n)-z_{2}(n)\right| \\
& +\left(a_{2}^{U} H_{1}+2 c_{2}^{U} H_{1}^{2}\right)\left|y_{1}\left(n-\tau_{1}\right)-z_{1}\left(n-\tau_{1}\right)\right| .
\end{aligned}
$$

Combining with (48) and calculating the $\Delta V$ of $V$ along the solution of (38), one has

$\Delta V(n)$

$$
\begin{aligned}
= & V(n+1)-V(n) \\
= & \left|y_{1}(n+1)-z_{1}(n+1)\right|+\left|y_{2}(n+1)-z_{2}(n+1)\right| \\
& +\sum_{\zeta=n+1-\tau_{1}}^{n}\left(a_{2}^{U} H_{1}+2 c_{2}^{U} H_{1}^{2}\right)\left|y_{1}(\zeta)-z_{1}(\zeta)\right| \\
& +\sum_{\zeta=n+1-\tau_{2}}^{n}\left(a_{1}^{U} H_{2}+2 c_{1}^{U} H_{2}^{2}\right)\left|y_{2}(\zeta)-z_{2}(\zeta)\right| \\
& -\left|y_{1}(n)-z_{1}(n)\right|-\left|y_{2}(n)-z_{2}(n)\right| \\
& -\sum_{\zeta=n-\tau_{1}}^{n-1}\left(a_{2}^{U} H_{1}+2 c_{2}^{U} H_{1}^{2}\right)\left|y_{1}(\zeta)-z_{1}(\zeta)\right|
\end{aligned}
$$

$$
-\sum_{\zeta=n-\tau_{2}}^{n-1}\left(a_{1}^{U} H_{2}+2 c_{1}^{U} H_{2}^{2}\right)\left|y_{2}(\zeta)-z_{2}(\zeta)\right|
$$$$
\leq\left|1-e^{\theta_{1}(n)}\right| \cdot\left|y_{1}(n)-z_{1}(n)\right|
$$$$
+\left(a_{1}^{U} H_{2}+2 c_{1}^{U} H_{2}^{2}\right)\left|y_{2}\left(n-\tau_{2}\right)-z_{2}\left(n-\tau_{2}\right)\right|
$$$$
+\left|1-e^{\theta_{2}(n)}\right| \cdot\left|y_{2}(n)-z_{2}(n)\right|
$$$$
+\left(a_{2}^{U} H_{1}+2 c_{2}^{U} H_{1}^{2}\right)\left|y_{1}\left(n-\tau_{1}\right)-z_{1}\left(n-\tau_{1}\right)\right|
$$$$
+\sum_{\zeta=n+1-\tau_{1}}^{n}\left(a_{2}^{U} H_{1}+2 c_{2}^{U} H_{1}^{2}\right)\left|y_{1}(\zeta)-z_{1}(\zeta)\right|
$$$$
+\sum_{\zeta=n+1-\tau_{2}}^{n}\left(a_{1}^{U} H_{2}+2 c_{1}^{U} H_{2}^{2}\right)\left|y_{2}(\zeta)-z_{2}(\zeta)\right|
$$$$
-\left|y_{1}(n)-z_{1}(n)\right|-\left|y_{2}(n)-z_{2}(n)\right|
$$$$
-\sum_{\zeta=n-\tau_{1}}^{n-1}\left(a_{2}^{U} H_{1}+2 c_{2}^{U} H_{1}^{2}\right)\left|y_{1}(\zeta)-z_{1}(\zeta)\right|
$$$$
-\sum_{\zeta=n-\tau_{2}}^{n-1}\left(a_{1}^{U} H_{2}+2 c_{1}^{U} H_{2}^{2}\right)\left|y_{2}(\zeta)-z_{2}(\zeta)\right|
$$

$$
\begin{aligned}
= & \left\{\left|1-e^{\theta_{1}(n)}\right|-1+\left(a_{2}^{U} H_{1}+2 c_{2}^{U} H_{1}^{2}\right)\right\} \cdot\left|y_{1}(n)-z_{1}(n)\right| \\
& +\left\{\left|1-e^{\theta_{2}(n)}\right|-1+\left(a_{1}^{U} H_{2}+2 c_{1}^{U} H_{2}^{2}\right)\right\} \cdot\left|y_{2}(n)-z_{2}(n)\right|
\end{aligned}
$$




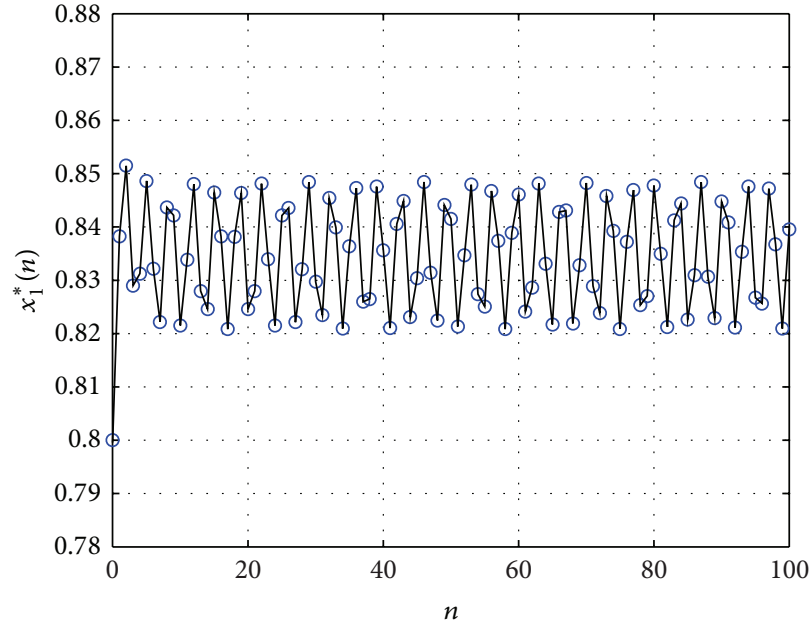

(a)

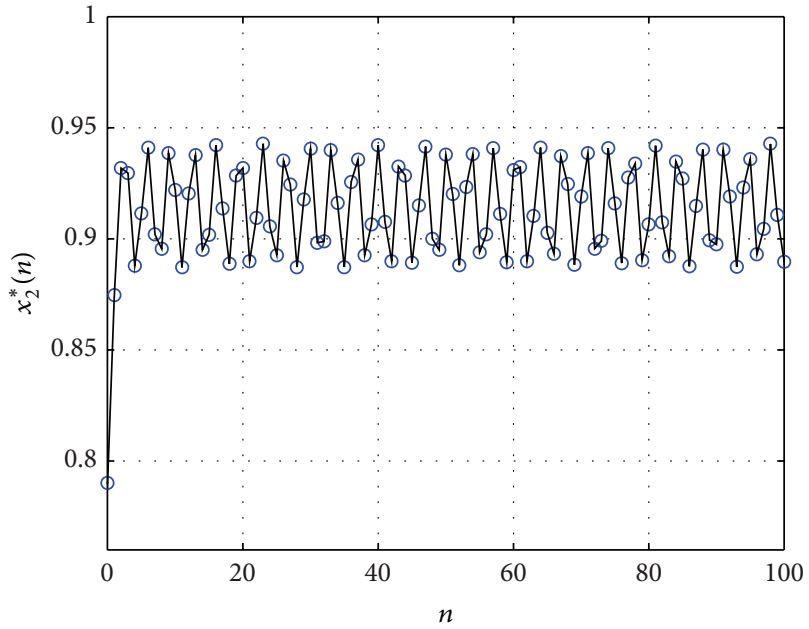

(b)

Figure 1: Positive almost periodic solution of system (50). (a) Time-series of $x_{1}^{*}(n)$ with initial values $x_{1}^{*}(-1)=0.79$ and $x_{1}^{*}(0)=0.80$ for $n \in[0,100]$. (b) Time-series of $x_{2}^{*}(n)$ with initial values $x_{2}^{*}(-2)=0.85, x_{2}^{*}(-1)=0.80$, and $x_{2}^{*}(0)=0.79$ for $n \in[0,100]$.

$$
\begin{aligned}
\leq & -\left\{1-\max \left(\left|1-h_{1}\right|,\left|1-H_{1}\right|\right)-\left(a_{2}^{U} H_{1}+2 c_{2}^{U} H_{1}^{2}\right)\right\} \\
& \cdot\left|y_{1}(n)-z_{1}(n)\right| \\
& -\left\{1-\max \left(\left|1-h_{2}\right|,\left|1-H_{2}\right|\right)-\left(a_{1}^{U} H_{2}+2 c_{1}^{U} H_{2}^{2}\right)\right\} \\
& \cdot\left|y_{2}(n)-z_{2}(n)\right| \\
= & -\lambda_{1}\left|y_{1}(n)-z_{1}(n)\right|-\lambda_{2}\left|y_{2}(n)-z_{2}(n)\right| \\
\leq & -\beta\left(\left|y_{1}(n)-z_{1}(n)\right|+\left|y_{2}(n)-z_{2}(n)\right|\right) \\
\leq & -\beta\left(\left(y_{1}(n)-z_{1}(n)\right)^{2}+\left(y_{2}(n)-z_{2}(n)\right)^{2}\right)^{1 / 2} \\
= & -\beta\left(\left|Y_{n}(0)-Z_{n}(0)\right|\right),
\end{aligned}
$$

where $\beta=\min \left\{\lambda_{1}, \lambda_{2}\right\}$. Since $\lambda_{i}>0, \beta>0$. Denote $c \in$ $C\left(R^{+}, R^{+}\right), c(x)=\beta x$; therefore, the condition in Remark 5 is satisfied. From Lemma 4 and Remark 5, system (32) has a unique uniformly asymptotically stable almost periodic solution denoted by $\left(y_{1}^{*}(n), y_{2}^{*}(n)\right)$, which is equivalent to saying that system (3) has a unique uniformly asymptotically stable positive almost periodic solution denoted by $\left(x_{1}^{*}(n), x_{2}^{*}(n)\right)$. This proof of Theorem 10 is completed.

If the coefficients $\left\{r_{i}(n)\right\},\left\{a_{i}(n)\right\}$, and $\left\{c_{i}(n)\right\}$ are bounded positive periodic sequences, then system (3) becomes a periodic version. Applying Lemma 4 and Theorem 10, Corollary 11 is obtained directly.

Corollary 11. Periodic system (3) shows a unique positive periodic solution which is uniformly asymptotically stable under the same assumptions of Theorem 10.

\section{Numerical Simulations}

In this section, we give two specific numerical examples to verify our analytical results, that is, Theorem 10 and Corollary 11.

Example 12. Consider the following delay discrete almost periodic competitive system:

$$
\begin{aligned}
& x_{1}(n+1) \\
& =x_{1}(n) \exp \left[(0.87+0.02 \sin (\sqrt{2} n \pi))-x_{1}(n)\right. \\
& -(0.025-0.001 \cos (\sqrt{2} n \pi)) x_{2}(n-2) \\
& \left.-(0.015+0.002 \sin (\sqrt{2} n \pi)) x_{2}^{2}(n-2)\right] \text {, } \\
& x_{2}(n+1) \\
& =x_{2}(n) \exp \left[(0.95+0.03 \cos (\sqrt{2} n \pi))-x_{2}(n)\right. \\
& -(0.020+0.001 \sin (\sqrt{3} n \pi)) x_{1}(n-1) \\
& \left.-(0.027+0.002 \sin (\sqrt{2} n \pi)) x_{1}^{2}(n-1)\right] \text {. }
\end{aligned}
$$

A computation shows that

$$
\begin{aligned}
& r_{1}^{U}=0.89, \quad r_{1}^{L}=0.85, \quad r_{2}^{U}=0.98, \quad r_{2}^{L}=0.92, \\
& a_{1}^{U}=0.026, \quad a_{1}^{L}=0.024, \quad a_{2}^{U}=0.021, \quad a_{2}^{L}=0.019 \text {, } \\
& c_{1}^{U}=0.017, \quad c_{1}^{L}=0.013, \quad c_{2}^{U}=0.029, \quad c_{2}^{L}=0.025 \text {, }
\end{aligned}
$$




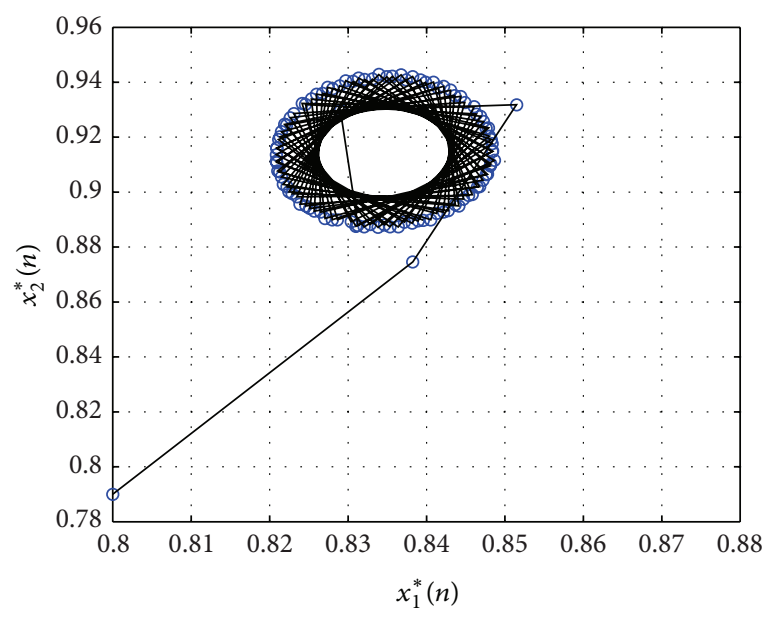

(a)

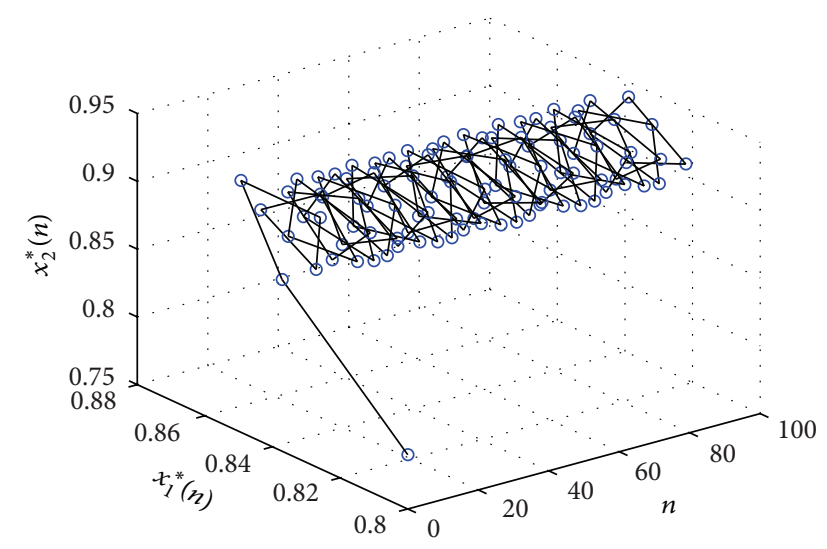

(b)

Figure 2: Phase portrait. (a) Two-dimensional phase portrait of $x_{1}^{*}(n)$ and $x_{2}^{*}(n)$ with initial values $x_{1}^{*}(-1)=0.79$ and $x_{1}^{*}(0)=0.80$ and $x_{2}^{*}(-2)=0.85, x_{2}^{*}(-1)=0.80$, and $x_{2}^{*}(0)=0.79$ for $n \in[0,100]$. (b) Three-dimensional phase portrait of $n, x_{1}^{*}(n)$ and $x_{2}^{*}(n)$ with the above initial values for $n \in[0,100]$.

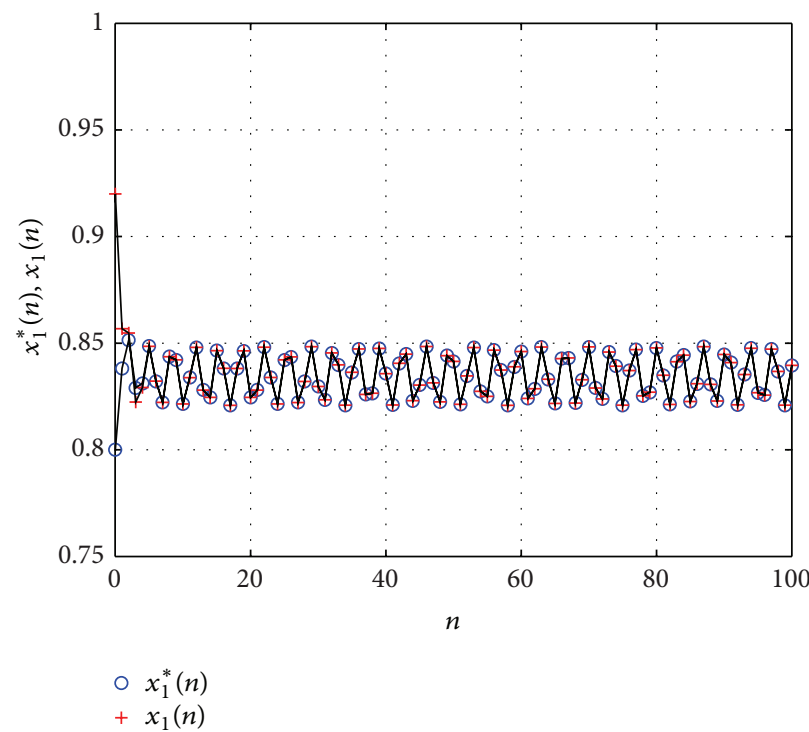

(a)

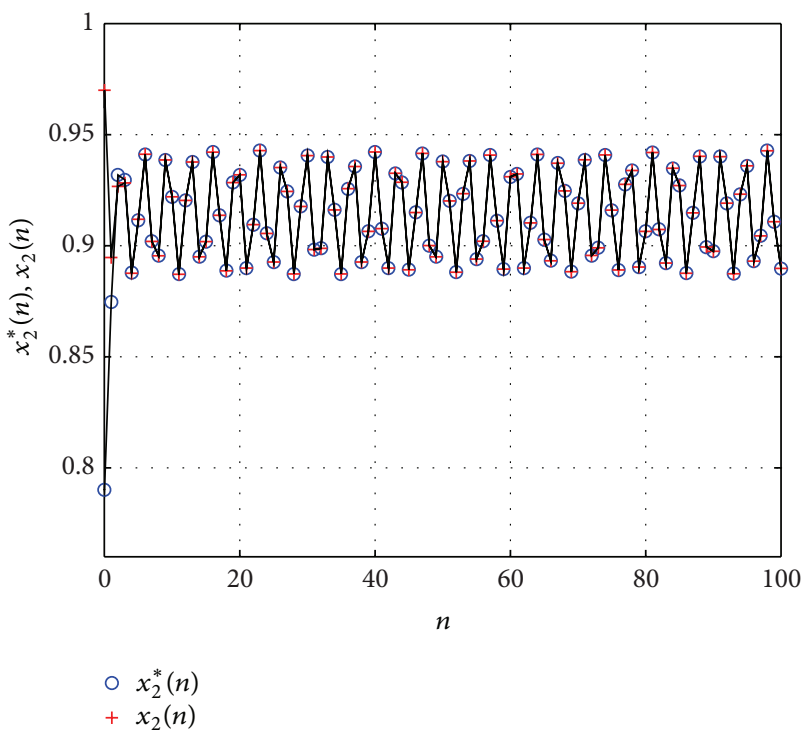

(b)

FIGURE 3: Uniformly asymptotic stability. (a) Time-series of $x_{1}^{*}(n)$ and $x_{1}(n)$ with initial values $x_{1}^{*}(-1)=0.79$ and $x_{1}^{*}(0)=0.80$ and $x_{1}(-1)=$ 0.83 and $x_{1}(0)=0.92$ for $n \in[0,100]$, respectively. (b) Time-series of $x_{2}^{*}(n)$ and $x_{2}(n)$ with initial values $x_{2}^{*}(-2)=0.85, x_{2}^{*}(-1)=0.80$, and $x_{2}^{*}(0)=0.79$ and $x_{2}(-2)=0.81, x_{2}(-1)=0.79$, and $x_{2}(0)=0.97$ for $n \in[0,100]$, respectively.

and then we have

$$
\begin{gathered}
H_{1} \approx 0.8958, \quad H_{2} \approx 0.9802, \quad \Delta_{1} \approx 0.8082, \\
\Delta_{2} \approx 0.8779, \quad h_{1} \approx 0.7404, \quad h_{2} \approx 0.7925, \\
\Delta_{1}>0, \quad \Delta_{2}>0 .
\end{gathered}
$$

Moreover,

$$
\begin{aligned}
\lambda_{1}= & 1-\max \left(\left|1-h_{1}\right|,\left|1-H_{1}\right|\right) \\
& -\left(a_{2}^{U} H_{1}+2 c_{2}^{U} H_{1}^{2}\right) \approx 0.6750>0,
\end{aligned}
$$

$$
\begin{aligned}
\lambda_{2}= & 1-\max \left(\left|1-h_{2}\right|,\left|1-H_{2}\right|\right) \\
& -\left(a_{1}^{U} H_{2}+2 c_{1}^{U} H_{2}^{2}\right) \approx 0.7343>0 .
\end{aligned}
$$

It is easy to see that the assumptions of Theorem 10 are satisfied; that is to say, system (50) has a unique positive almost periodic solution denoted by $\left(x_{1}^{*}(t), x_{2}^{*}(t)\right)$ which is uniformly asymptotically stable (see Figure 1 ), and the two-dimensional and three-dimensional phase portraits are displayed in Figure 2, respectively. In Figure 3, any positive 


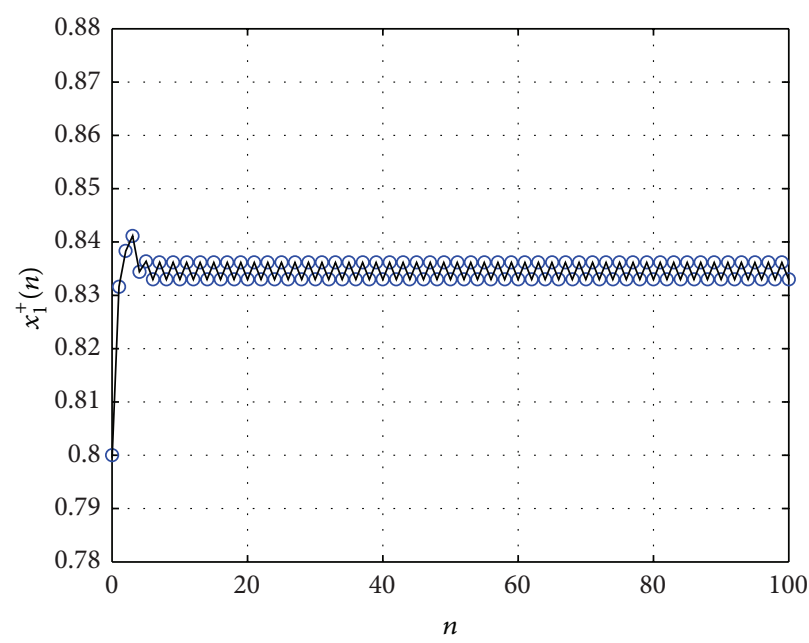

(a)

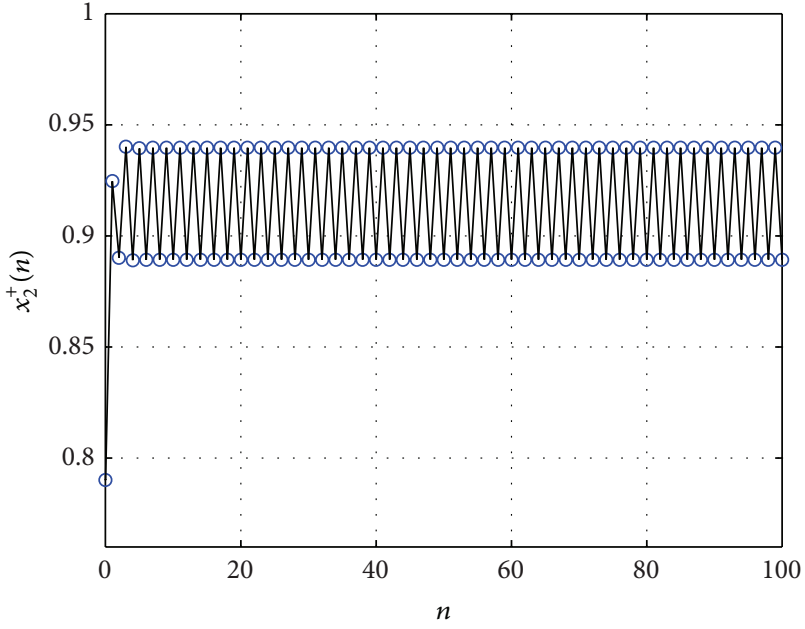

(b)

FIGURE 4: Positive periodic solution of system (54). (a) Time-series of $x_{1}^{+}(n)$ with initial values $x_{1}^{+}(-1)=0.79$ and $x_{1}^{+}(0)=0.80$ for $n \in[0,100]$. (b) Time-series of $x_{2}^{+}(n)$ with initial values $x_{2}^{+}(-2)=0.85, x_{2}^{+}(-1)=0.80$, and $x_{2}^{+}(0)=0.79$ for $n \in[0,100]$.

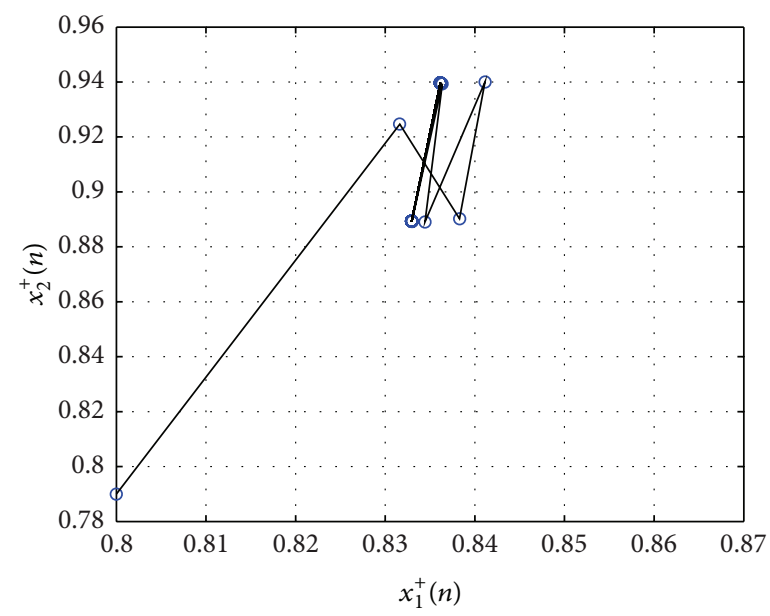

(a)

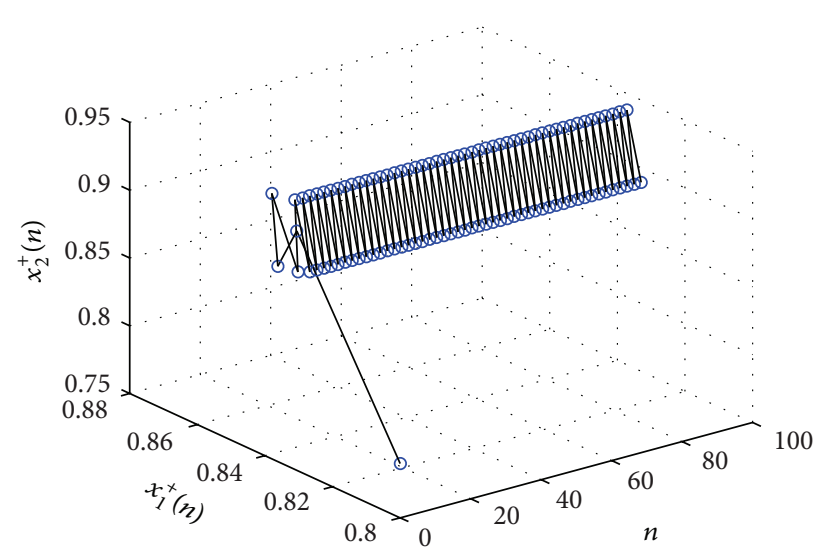

(b)

FIGURE 5: Phase portrait. (a) Two-dimensional phase portrait of $x_{1}^{+}(n)$ and $x_{2}^{+}(n)$ with initial values $x_{1}^{+}(-1)=0.79$ and $x_{1}^{+}(0)=0.80$ and $x_{2}^{+}(-2)=0.85, x_{2}^{+}(-1)=0.80$, and $x_{2}^{+}(0)=0.79$ for $n \in[0,100]$. (b) Three-dimensional phase portrait of $n, x_{1}^{+}(n)$ and $x_{2}^{+}(n)$ with the above initial values for $n \in[0,100]$.

solution denoted by $\left(x_{1}(n), x_{2}(n)\right)$ tends to the above almost periodic solution $\left(x_{1}^{*}(n), x_{2}^{*}(n)\right)$.

Example 13. Consider the following delay discrete periodic competitive system:

$$
\begin{aligned}
& x_{1}(n+1) \\
& \begin{aligned}
=x_{1}(n) \exp [ & (0.87+0.02 \sin (n \pi))-x_{1}(n) \\
& -(0.025-0.001 \cos (n \pi)) x_{2}(n-2) \\
& \left.-(0.015+0.002 \sin (n \pi)) x_{2}^{2}(n-2)\right],
\end{aligned}
\end{aligned}
$$

$$
\begin{aligned}
& x_{2}(n+1) \\
& \begin{aligned}
=x_{2}(n) \exp [ & (0.95+0.03 \cos (n \pi))-x_{2}(n) \\
& -(0.020+0.001 \sin (n \pi)) x_{1}(n-1) \\
& \left.-(0.027+0.002 \sin (n \pi)) x_{1}^{2}(n-1)\right] .
\end{aligned}
\end{aligned}
$$

Analogously, we can see that system (54) satisfies the assumptions of Theorem 10. From Corollary 11, there exists a unique positive periodic solution of system (54) which is uniformly asymptotically stable. From Figure 4 , system (54) shows a positive periodic solution denoted by $\left(x_{1}^{+}(t), x_{2}^{+}(t)\right)$, 


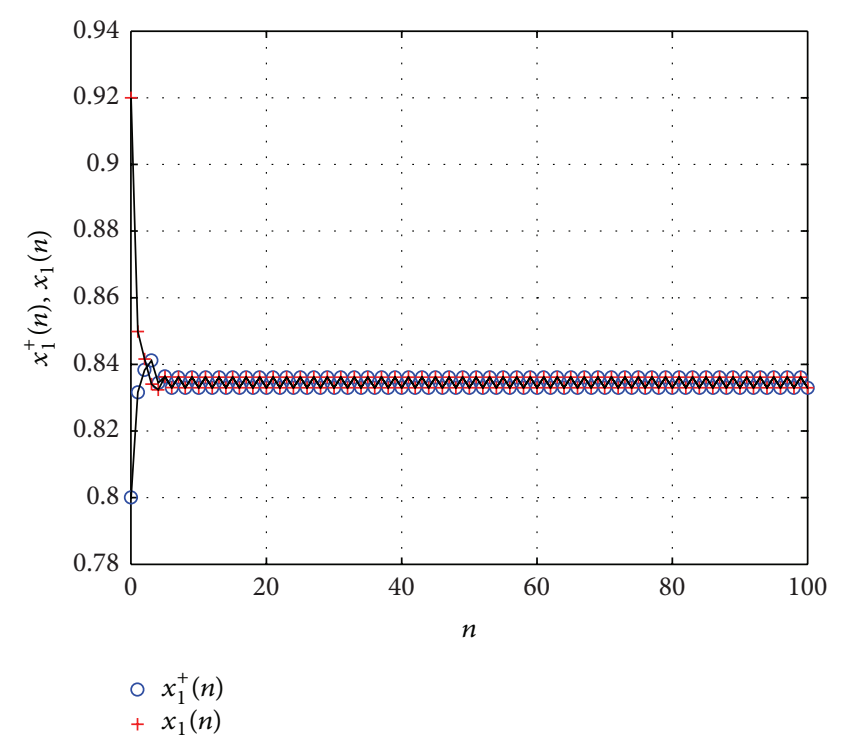

(a)

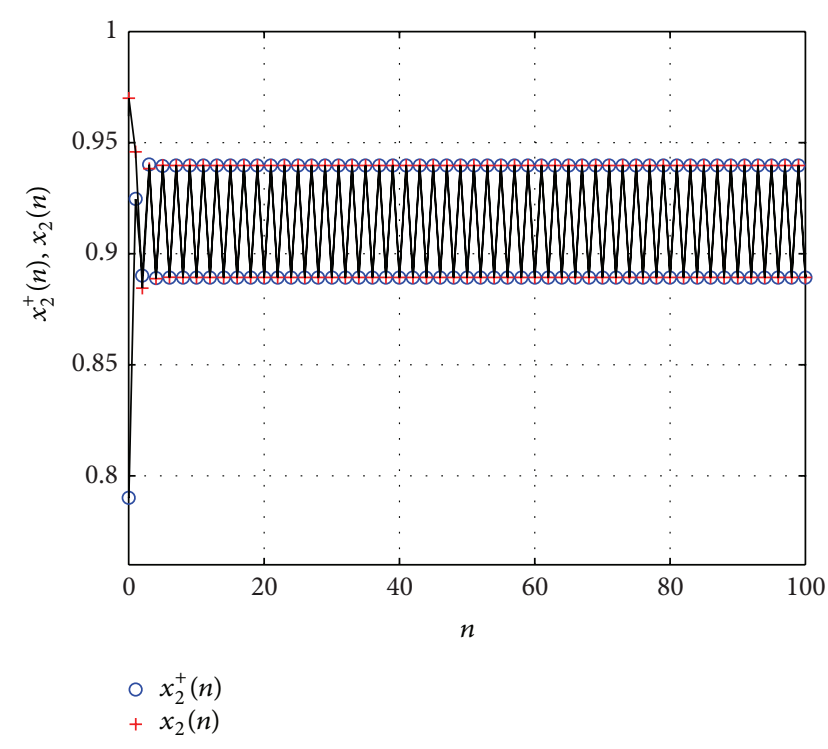

(b)

FIGURE 6: Uniformly asymptotic stability. (a) Time-series of $x_{1}^{+}(n)$ and $x_{1}(n)$ with initial values $x_{1}^{+}(-1)=0.79$ and $x_{1}^{+}(0)=0.80$ and $x_{1}(-1)=$ 0.83 and $x_{1}(0)=0.92$ for $n \in[0,100]$, respectively. (b) Time-series of $x_{2}^{+}(n)$ and $x_{2}(n)$ with initial values $x_{2}^{+}(-2)=0.85, x_{2}^{+}(-1)=0.80$, and $x_{2}^{+}(0)=0.79$ and $x_{2}(-2)=0.81, x_{2}(-1)=0.79$, and $x_{2}(0)=0.97$ for $n \in[0,100]$, respectively.

and the two-dimensional and three-dimensional phase portraits are displayed in Figure 5, respectively. Figure 6 shows that any positive solution denoted by $\left(x_{1}(n), x_{2}(n)\right)$ tends to the above periodic solution $\left(x_{1}^{+}(n), x_{2}^{+}(n)\right)$.

\section{Conflict of Interests}

The authors declare that there is no conflict of interests regarding the publication of this paper.

\section{Acknowledgments}

The authors would like to thank the referees for their valuable comments that greatly improved the presentation of this paper. They are also grateful to Professor Zhijun Liu for his suggestions and doctoral student Qinglong Wang for his help in numerical simulations.

\section{References}

[1] Z. Liu and L. Chen, "Positive periodic solution of a general discrete non-autonomous difference system of plankton allelopathy with delays," Journal of Computational and Applied Mathematics, vol. 197, no. 2, pp. 446-456, 2006.

[2] R. P. Agarwal, Difference Equations and Inequalities: Theory, Methods and Applications, vol. 228 of Monographs and Textbooks in Pure and Applied Mathematics, Marcel Dekker, New York, NY, USA, 2nd edition, 2000.

[3] J. D. Murray, Mathematical Biology, Springer, New York, NY, USA, 1989.

[4] Z. Zhou and X. Zou, "Stable periodic solutions in a discrete periodic logistic equation," Applied Mathematics Letters, vol. 16, no. 2, pp. 165-171, 2003.
[5] M. Fan and K. Wang, "Periodic solutions of a discrete time nonautonomous ratio-dependent predator-prey system," Mathematical and Computer Modelling, vol. 35, no. 9-10, pp. 951-961, 2002.

[6] W. D. Wendi, G. Mulone, F. Salemi, and V. Salone, "Global stability of discrete population models with time delays and fluctuating environment," Journal of Mathematical Analysis and Applications, vol. 264, no. 1, pp. 147-167, 2001.

[7] R. Xu, M. A. Chaplain, and F. A. Davidson, "Periodic solutions of a discrete nonautonomous Lotka-Volterra predator-prey model with time delays," Discrete and Continuous Dynamical Systems Series B, vol. 4, no. 3, pp. 823-831, 2004.

[8] Q. L. Wang and Z. J. Liu, "Uniformly asymptotic stability of almost periodic solutions for a delay difference system of plankton allelopathy," Advances in Difference Equations, vol. 2013, article 283, 14 pages, 2013.

[9] Y. Li and T. Zhang, "Almost periodic solution for a discrete hematopoiesis model with time delay," International Journal of Biomathematics, vol. 5, no. 1, Article ID 1250003, 1250003, 9 pages, 2012.

[10] Z. Li, F. Chen, and M. He, "Almost periodic solutions of a discrete Lotka-Volterra competition system with delays," Nonlinear Analysis: Real World Applications, vol. 12, no. 4, pp. 23442355, 2011.

[11] Y. Song, "Positive almost periodic solutions of nonlinear discrete systems with finite delay," Computers \& Mathematics with Applications, vol. 58, no. 1, pp. 128-134, 2009.

[12] S. Zhang and G. Zheng, "Almost periodic solutions of delay difference systems," Applied Mathematics and Computation, vol. 131, no. 2-3, pp. 497-516, 2002.

[13] C. Niu and X. Chen, "Almost periodic sequence solutions of a discrete Lotka-Volterra competitive system with feedback control," Nonlinear Analysis: Real World Applications, vol. 10, no. 5, pp. 3152-3161, 2009. 
[14] T. Zhang, Y. Li, and Y. Ye, "Persistence and almost periodic solutions for a discrete fishing model with feedback control," Communications in Nonlinear Science and Numerical Simulation, vol. 16, no. 3, pp. 1564-1573, 2011.

[15] F. J. Ayala, M. E. Gilpin, and J. G. Ehrenfeld, "Competition between species: theoretical models and experimental tests," Theoretical Population Biology, vol. 4, pp. 331-356, 1973.

[16] K. Gopalsamy, Stability and Oscillations in Delay Differential Equations of Population Dynamics, Kluwer Academic, Dordrecht, The Netherlands, 1992.

[17] Y. Rong and H. Jialin, "The existence of almost periodic solutions for a class of differential equations with piecewise constant argument," Nonlinear Analysis: Theory, Methods \& Applications, vol. 28, no. 8, pp. 1439-1450, 1997.

[18] X. Yang, "Uniform persistence and periodic solutions for a discrete predator-prey system with delays," Journal of Mathematical Analysis and Applications, vol. 316, no. 1, pp. 161-177, 2006. 


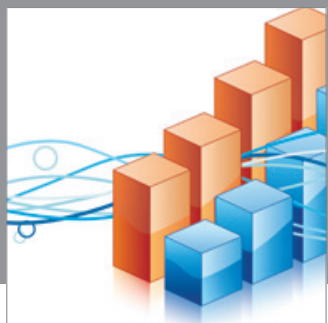

Advances in

Operations Research

mansans

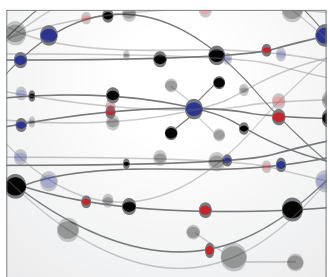

The Scientific World Journal
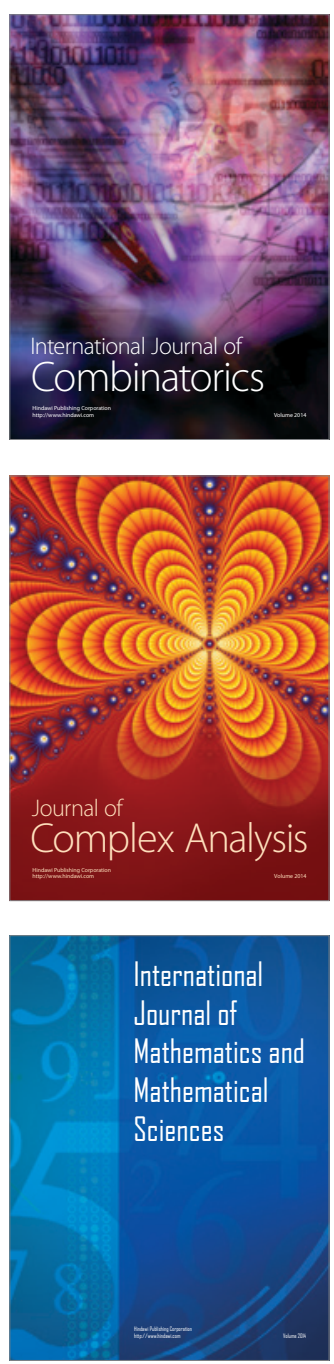
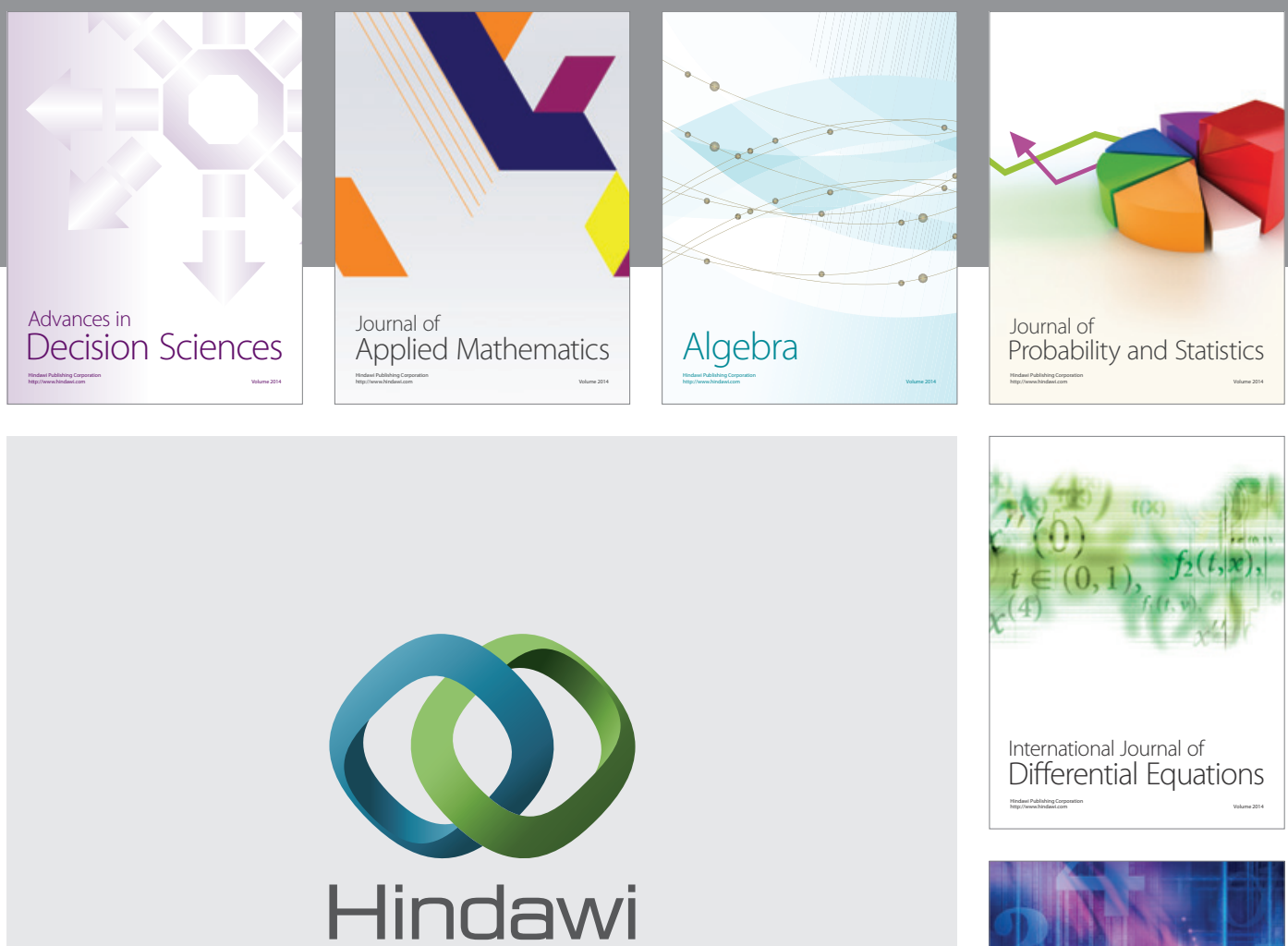

Submit your manuscripts at http://www.hindawi.com
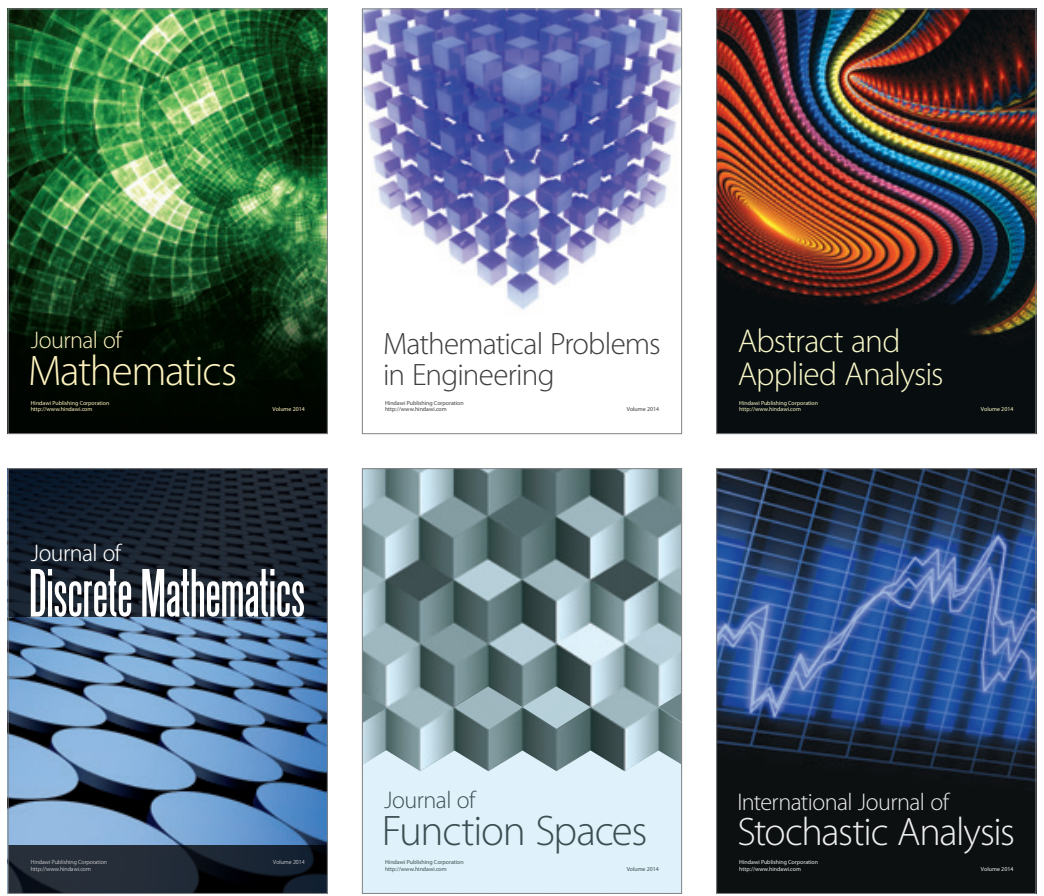

Journal of

Function Spaces

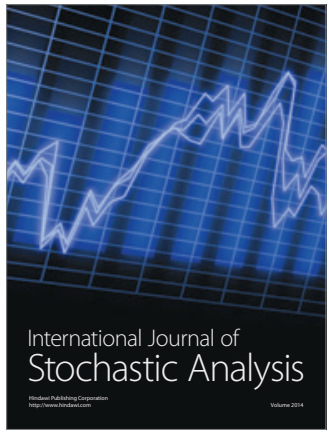

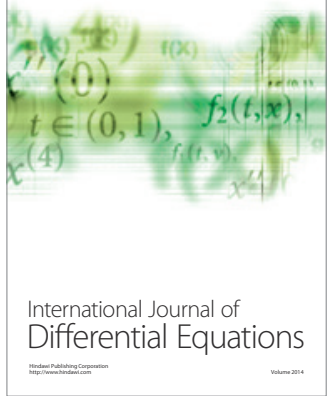
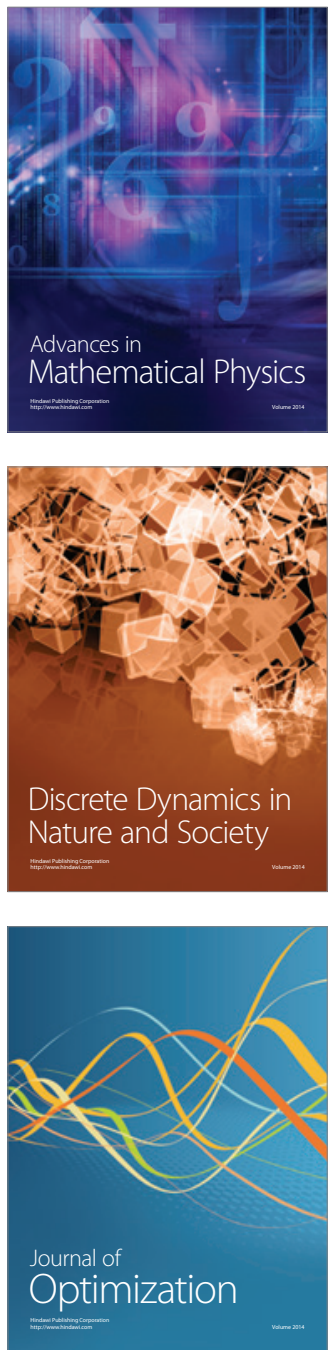